\title{
Mechanism of the Far-Infrared Absorption of Carbon-Nanotube Films
}

\author{
T. Kampfrath, ${ }^{1}$ K. von Volkmann, ${ }^{1}$ C. M. Aguirre, ${ }^{2}$ P. Desjardins,${ }^{2}$ R. Martel,,${ }^{3}$ M. Krenz, ${ }^{1}$ C. Frischkorn, ${ }^{1}$ \\ M. Wolf, ${ }^{1}$ and L. Perfetti ${ }^{1}$ \\ ${ }^{1}$ Fachbereich Physik, Freie Universität Berlin, 14195 Berlin, Germany \\ ${ }^{2}$ Département de Génie Physique, École Polytechnique, Montréal, Canada H3C-3A7 \\ ${ }^{3}$ Département de Chimie, Université de Montréal, Montréal, Canada H3C-3J7
}

(Received 10 June 2008; published 29 December 2008)

\begin{abstract}
The far-infrared conductivity of single-wall carbon-nanotube ensembles is dominated by a broad absorption peak around $4 \mathrm{THz}$ whose origin is still debated. We observe an overall depletion of this peak when the nanotubes are excited by a short visible laser pulse. This finding excludes optical absorption due to a particle-plasmon resonance and instead shows that interband transitions in tubes with an energy gap of $\sim 10 \mathrm{meV}$ dominate the far-infrared conductivity. A simple model based on an ensemble of two-level systems naturally explains the weak temperature dependence of the far-infrared conductivity by the tubeto-tube variation of the chemical potential.
\end{abstract}

DOI: 10.1103/PhysRevLett.101.267403

A single-wall carbon nanotube (NT) can be pictured as a rolled-up hexagonal sheet of carbon atoms where diameter and chirality determine whether the tube is semiconducting or truly metallic [1]. The electronic energy gap of semiconducting tubes with a diameter of $1 \mathrm{~nm}$ lies in the $10 \mathrm{meV}$ or $1 \mathrm{eV}$ range for small- and large-gap tubes, respectively [2]. In the framework of a tight-binding model, the large gaps arise from the quantization of the electron wave vector along the circumference of the NT whereas the small gaps are due to the curvature of the NT wall along this direction. In addition, the interaction between the NT and its local environment, such as other tubes or adsorbed molecules, can cause the opening of a small energy gap [3]. Because of this high sensitivity of the electronic structure to environmental changes along with the large tube surface, NTs have been suggested as effective chemical sensors [4].

The optical properties of NTs in the visible spectral range are well understood: As unveiled by complementary nonlinear-optical techniques, the absorption of visible light leads to the generation of excitons in the large-gap tubes [5-7]. In contrast, the microscopic mechanism of the farinfrared (FIR) absorption is still unclear: The broad absorption feature at about $4 \mathrm{THz}$ was first assigned to optical interband transitions across the electronic energy gap of small-gap NTs as illustrated in Fig. 1(a) [8]. However, for an electronic transition with such low energy, this peak shows a surprisingly weak temperature dependence [8]. As a consequence, phonons [9] and, as sketched in Fig. 1(b), particle plasmons [10] were suggested as alternative mechanisms. It is crucial to clarify this point, since the FIR absorption has already been extensively used as a sensitive probe of side chains covalently bound to the NT wall [11] and of the doping level of the tubes [12-14].

In this Letter, we show that optical transitions across the energy gap of small-gap tubes dominate the FIR conductivity of NTs. First, photoexcitation of the NTs induces an overall drop but no shift of the FIR conductivity peak,
PACS numbers: 78.67.Ch, 72.80.Rj, 73.63.Fg, 78.47.-p

which excludes a plasmonic resonance as an absorption mechanism. Such behavior is rather a clear fingerprint of optical interband transitions blocked by electron-hole pairs. Second, a simple model based on an ensemble of two-level systems naturally explains the weak temperature dependence of the FIR absorption by the tube-to-tube variation of the chemical potential, a mechanism that has not been considered so far.

Our sample is a $700 \mathrm{~nm}$ thick film of high-pressure-COgrown NTs on a diamond substrate, and its preparation and characterization are detailed elsewhere [15]. The NTs in our sample are partially bundled, and their diameter distribution extends from 0.8 to $1.2 \mathrm{~nm}$ [16]. Atomic-force micrographs suggest a NT space-filling fraction $\mathcal{F}$ of $\sim 0.2$. Roughly $2 / 3$ of all NTs are large-gap tubes which become transparent at photon energies below $0.6 \mathrm{eV}$, whereas the small-gap and metallic NTs can still absorb photons [17].

For the FIR conductivity measurements, we employ a $\mathrm{THz}$ spectrometer driven by laser pulses of $10 \mathrm{fs}$ duration, $790 \mathrm{~nm}$ center wavelength, $10 \mathrm{~nJ}$ energy, and $75 \mathrm{MHz}$ repetition rate from a Ti:sapphire laser oscillator
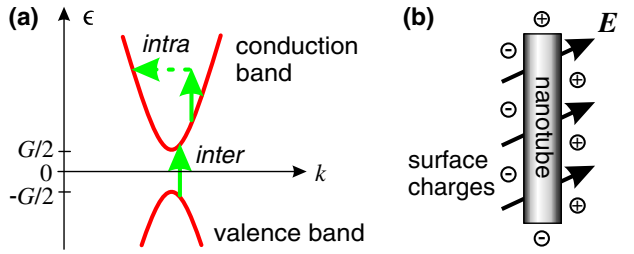

FIG. 1 (color online). Schematic of proposed mechanisms explaining the far-infrared absorption of NTs. (a) Interband transitions across the band gap $G$ of a small-gap NT. An impurity-assisted intraband transition in the band structure $\boldsymbol{\epsilon}(k)$ is also shown. (b) Particle-plasmon resonance. The probing electric field $\boldsymbol{E}$ induces charges on the NT surface which lead to an additional repulsive force on the electrons inside. 
(Femtolasers M1). Part of the laser output is focused onto a $\mathrm{ZnTe}, \mathrm{GaP}$, or GaSe crystal to generate ultrashort $\mathrm{THz}$ pulses by difference-frequency mixing [18]. After transmission through the NT sample, the THz electric field is detected electro-optically by a sampling pulse in a ZnTe or $\mathrm{GaP}$ crystal $[19,20]$. Our spectrometer covers the frequency window from 1 to $40 \mathrm{THz}$ by using various pairs of emitter and detection crystals, see Fig. 2(a). The lowtemperature measurements employ a cryostat (Cryovac Konti) whereas high electronic temperatures are realized by an additional laser pulse which heats the sample prior to probing. For this pump-probe configuration, the THz spectrometer is driven by $20 \mathrm{fs}, 800 \mathrm{~nm}, 600 \mu \mathrm{J}, 1 \mathrm{kHz}$ pulses from an amplified Ti:sapphire laser system (Femtolasers Femtopower Pro).

We detect $\mathrm{THz}$ pulses transmitted through the diamond substrate only, an unexcited sample, and a sample excited at some delay $\tau$ before arrival of the $\mathrm{THz}$ probe. By applying Fresnel formulas to these data, we obtain the conductivity $\sigma_{\infty}$ of the unexcited sample and the pumpinduced changes $\Delta \sigma_{\tau}=\sigma_{\tau}-\sigma_{\infty}$. Whenever convenient, we also refer to the dielectric function $\varepsilon_{\tau}=1-$ $c Z_{0} \sigma_{\tau} / \mathrm{i} \omega$ where $Z_{0} \approx 377 \Omega$ is the vacuum impedance.

Figures 2(a) and 2(b) show $\operatorname{Re} \sigma_{\infty}(\omega)$ and $\operatorname{Re} \varepsilon_{\infty}(\omega)$ of the unexcited sample. $\operatorname{Re} \sigma_{\infty}(\omega)$ quantifies how strongly the sample absorbs light of frequency $\omega / 2 \pi$. It exhibits a

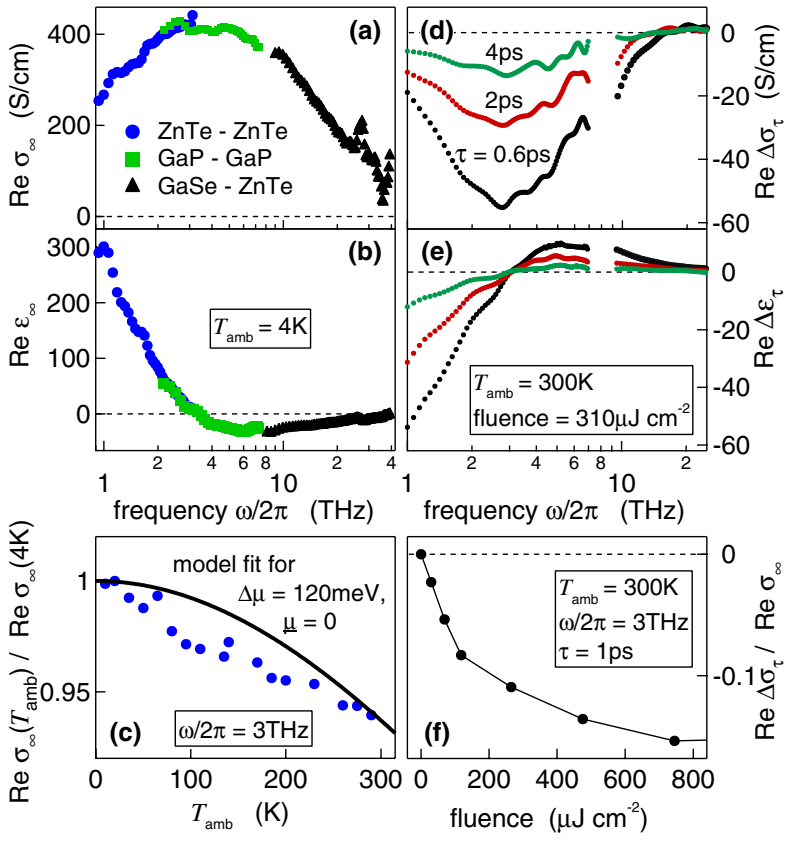

FIG. 2 (color online). Experimentally determined FIR conductivity of the $(\mathrm{a}-\mathrm{c})$ unexcited and $(\mathrm{d}-\mathrm{f})$ excited NT sample. (a) Real part of the equilibrium conductivity. Different symbols indicate different combinations of $\mathrm{THz}$-generation and detection crystals. (b) Corresponding real part of the dielectric function. (c) $\operatorname{Re} \sigma_{\infty}$ as a function of ambient temperature $T_{\text {amb }}$ together with a fit according to Eq. (3). (d, e) Pump-induced changes in the sample conductivity and dielectric function at several pumpprobe delays $\tau$. (f) $\operatorname{Re} \Delta \sigma_{\tau}$ as a function of the pump fluence. broad peak centered at a resonance frequency $\omega_{\text {res }} / 2 \pi$ of roughly $4 \mathrm{THz}$ in agreement with previous reports [814,21]. As seen in Fig. 2(c), the FIR absorption peak decreases only by less than $10 \%$ when $T_{\text {amb }}$ is raised from 4 to $300 \mathrm{~K}$. A further reduction of the conductivity peak occurs when the sample is heated by a pump pulse, which is demonstrated by the negative $\operatorname{Re} \Delta \sigma_{\tau}$ for several pump-probe delays $\tau$ in Fig. 2(d). Figures 2(d) and 2(e) show also that the pump-induced signal decays uniformly on a time scale of several picoseconds. Finally, as seen in Fig. 2(f), $\left|\operatorname{Re} \Delta \sigma_{\tau}\right|$ increases with the incident pump fluence for fluences below $100 \mu \mathrm{J} \mathrm{cm}^{-2}$ but saturates at higher fluences. Above a fluence of $\approx 1 \mathrm{~mJ} \mathrm{~cm}^{-2}$, we find that the NTs are damaged irreversibly.

Two observations suggest that optical transitions across the energy gap $G$ of the small-gap tubes, as sketched in Fig. 1(a), dominate the FIR conductivity of NT ensembles: First, the photon energy $\hbar \omega_{\text {res }} \approx 16 \mathrm{meV}$ at the absorption maximum in Fig. 2(a) is compatible with the energy gap $G$ of individual small-gap NTs that were determined by scanning tunneling spectroscopy [2]. Second, $\hbar \omega_{\text {res }}$ increases when the mean tube diameter of a NT ensemble decreases and, thus, the mean $G$ increases [2,10,14]. However, Fig. 2(c) implies that the temperature dependence of electronic transitions with such low energy would be puzzlingly weak. Consequently, alternative mechanisms such as infrared-active phonons [9] and particle plasmons [10] were suggested to cause the broad FIR absorption feature. We exclude the phonon scenario since theory neither predicts broad phonon bands nor do the vibrating carbon atoms produce large electric dipole moments required for an effective absorption of $\mathrm{THz}$ radiation [21].

As to the plasmon scenario, it is important to realize that the measured $\sigma_{\infty}$ shown in Figs. 2(a) and 2(b) is the conductivity of an effective and homogeneous medium since our NT film is uniform on the $\sim 10 \mu \mathrm{m}$ scale of the probe wavelength. More explicitly, effective-medium theory in Maxwell-Garnett approximation yields [22]

$$
\varepsilon=\varepsilon^{\mathrm{h}}+\frac{1}{3} \sum_{s} \mathcal{F}^{s}\left[\varepsilon_{\|}^{s}-\varepsilon^{\mathrm{h}}+4 \varepsilon^{\mathrm{h}} \frac{\varepsilon_{\perp}^{s}-\varepsilon^{\mathrm{h}}}{\varepsilon_{\perp}^{s}+\varepsilon^{\mathrm{h}}}\right]
$$

where $\mathcal{F}^{s}$ is the space-filling fraction of tube sort $s$ with dielectric function $\varepsilon_{\|}^{s}$ parallel and $\varepsilon_{\perp}^{s}$ perpendicular to the tube axis. $\varepsilon^{\mathrm{h}} \approx 1$ is the dielectric function of the host medium. The effective medium will show enhanced absorption at frequencies with minimum denominator $\varepsilon_{\perp}^{s}+$ $\varepsilon^{\text {h }}$ in Eq. (1). The mechanism behind such particleplasmon resonance is illustrated in Fig. 1(b): The probing electric field $\boldsymbol{E}$ induces charges on the NT surface which partially compensate the external field inside the tube. This so-called depolarization field acts as an additional repulsive force on the NT electrons resulting in a blueshift of the "bare" resonances of $\varepsilon_{\perp}^{s}$. Since a NT is typically 100 times longer than wide, the depolarization field is much smaller along the tube axis than perpendicular to it. This is con- 
sistent with Eq. (1) where only the $\varepsilon_{\perp}^{s}$ but not the $\varepsilon_{\|}^{s}$ show up in the resonance denominator.

Let us now assume that the broad peak in Fig. 2(a) was due to a plasmon resonance. An elegant way to check the validity of this assumption is to excite the sample with a visible pump pulse, such that the $\varepsilon_{\perp}^{s}$ undergo transient changes. Equation (1) then implies a shift of the peak in Fig. 2(a) and, thus, a derivativelike structure of the pumpinduced changes $\operatorname{Re} \Delta \sigma_{\tau}$. As an example, such transient blueshift was observed for GaAs nanowires in which the pump pulse increased the plasma frequency [23]. Our pump-probe data in Fig. 2(d), however, do not show any change in sign below $15 \mathrm{THz}$ thus clearly excluding a particle-plasmon based absorption mechanism. Equation (1) then suggests that the pump-induced conductivity changes are dominated by the $\|$ component, that is $\varepsilon \approx$ $(1-\mathcal{F} / 3) \varepsilon^{\mathrm{h}}+\sum \mathcal{F}^{s} \varepsilon_{\|}^{s} / 3$ where $\mathcal{F}=\sum \mathcal{F}^{s}$. We emphasize that this finding agrees with FIR absorption measurements on aligned NTs [10] where for $\mathrm{THz}$ radiation polarized parallel to the tube axes the usual FIR absorption peak was observed. It vanished for perpendicular polarization, totally contrary to the behavior expected from a particle-plasmon resonance.

The following microscopic model shows that the overall reduction of the FIR absorption peak of the pump-excited sample is a clear fingerprint of interband transitions blocked by transient hot electrons. Given that the model of independent electrons is valid in the small-gap tubes, the Fermi function $f_{\mu T}(\epsilon)=\left\{\exp \left[(\epsilon-\mu) / k_{\mathrm{B}} T\right]+1\right\}^{-1}$ describes the occupation of a single-electron Bloch state with energy $\epsilon$. Here, the chemical potential $\mu$ is determined by the band structure, the electronic temperature $T$, and the number of electrons within an individual NT. Note that $\mu$ may vary from tube to tube since we have fixed the $\epsilon$ origin at the gap center for each tube. Figure 1(a) sketches the band structure of a small-gap NT with band-gap energy $G$ along the one-dimensional electron wave vector $k$ together with optical transitions.

An electron absorbs a probing $\mathrm{THz}$ photon by an intraband or an interband optical transition. All transitions may be assisted by electron scattering off lattice defects, phonons, or other electrons [24,25]. Intraband transitions permit arbitrarily low excitation energies and lead to a Drudelike contribution, which is characterized by large negative values of $\operatorname{Re} \varepsilon_{\infty}$. Since such behavior is not observed in our data in Fig. 2(b), only interband transitions are considered further. They mainly connect states near the top of the valence band and the bottom of the conduction band where the density of states is the highest. Moreover, tight-binding calculations predict that the transition matrix elements are dominant for precisely these transitions [26]. The strength of a transition in the resulting two-level scheme is proportional to the difference

$$
F_{\mu T G}=f_{\mu T}(-G / 2)-f_{\mu T}(G / 2)
$$

of the final- and initial-state occupation numbers. The real part of the conductivity of a single NT is then $C \delta(\hbar \omega-$ $G) F_{\mu T G} / \omega[24,25,27]$. The prefactor $C$ contains all transition matrix elements connecting the band-edge states and is assumed to be independent of the NT type.

As outlined above, the effective conductivity is approximately obtained by summing up the $\|$ conductivities of all NTs. Equivalently, one can also integrate over all values of $\mu, T$, and $G$ weighted with the combined probability distribution $p_{\mu T G}$. We ignore possible correlations among these quantities, $p_{\mu T G}=p_{\mu} p_{T} p_{G}$, and assume one and the same electronic temperature $T$ in all tubes. The integration then reduces to

$$
\operatorname{Re} \sigma(\omega) \propto \frac{p_{G}}{3 \omega} \int \mathrm{d} \mu p_{\mu} F_{\mu T G}=: \frac{p_{G}}{3 \omega}\left\langle F_{\mu T G}\right\rangle
$$

where $G=\hbar \omega$. The real part $\operatorname{Re} \varepsilon$ of the dielectric function is obtained by a Hilbert transformation of $\operatorname{Re} \sigma$.

Comparing the spectral behavior of Eq. (3) to that of the measured equilibrium conductivity in Fig. 2(a) requires the probability distributions $p_{G}$ and $p_{\mu}$ of the gap energy and the chemical potential, respectively. Since those are a priori unknown we prefer to compare the temperature dependence of $\operatorname{Re} \sigma$ at a fixed frequency of $3 \mathrm{THz}$, which merely requires knowledge of $p_{\mu}$. Assuming a Gaussian distribution $p_{\mu} \propto \exp \left[-(\mu-\mu)^{2} / 2 \Delta \mu^{2}\right]$, we obtain the $\operatorname{Re} \sigma$-versus- $T$ curves displayed in Fig. 3(a) for $\mu=0$ and various standard deviations $\Delta \mu$.

In case of a sharply defined chemical potential, $\Delta \mu=0$, our model predicts a strong decrease of the conductivity with increasing temperature. Such a decrease, however, is not observed in the experimental data of Fig. 2(c). On the other hand, increasing $\Delta \mu$ gradually weakens the temperature dependence of $\operatorname{Re} \sigma$ as seen in Fig. 3(a). Finally, at $\Delta \mu=120 \mathrm{meV}$, the modeled $\operatorname{Re} \sigma$-versus- $T$ curve matches the measured curve of Fig. 2(c) quite well. The reason for this behavior is illustrated by Fig. 3(b): When the Fermi functions in Eq. (3) are convoluted with a $\mu$ distribution of width $\Delta \mu \gg k_{\mathrm{B}} T$, the slope of the Fermi edge is reduced so strongly that the averaged occupationnumber difference $\left\langle F_{\mu T G}\right\rangle$ becomes nearly independent of temperature. It has to be emphasized that $\Delta \mu \neq 0$ rather than $\mu \neq 0$ is the crucial condition to weaken the temperature dependence of the FIR conductivity [28]. We conclude that the weak temperature dependence of the FIR conductivity is consistent with a tube-to-tube variation of the chemical potential of about $0.1 \mathrm{eV}$ in our sample. This value is in good agreement with the $\mu$ variations found by conductivity measurements of individual small-gap NTs [29] and Raman spectroscopy of large-gap NT bundles [30]. Possible origins of the $\mu$ variation are spurious doping and charge transfer between NTs with differing work function [31].

The validity of our interpretation can be further tested by analyzing the transient changes in the FIR conductivity when the sample is excited by an ultrashort visible pump pulse. The $\operatorname{Re} \Delta \sigma_{\tau}$ and $\operatorname{Re} \Delta \varepsilon_{\tau}$ are shown in Figs. 2(d) and 


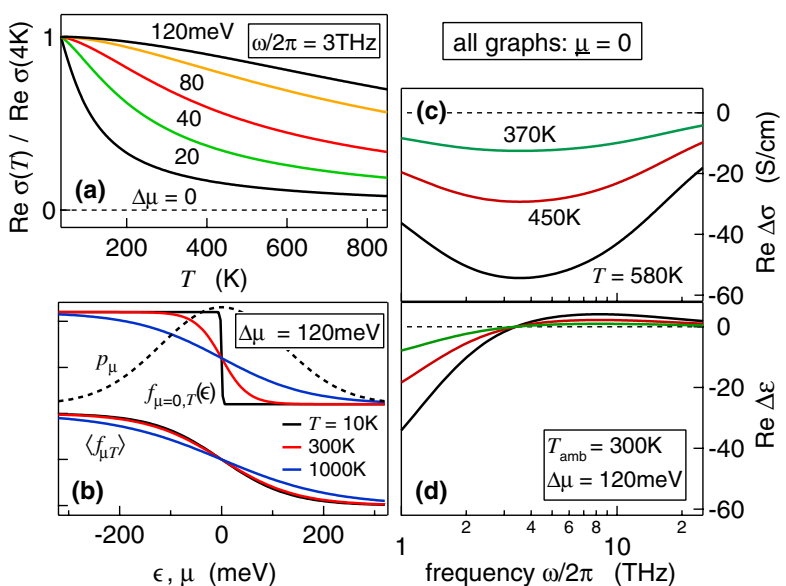

FIG. 3 (color online). Theoretical results for the FIR conductivity of a NT ensemble. (a) Modeled $\operatorname{Re} \sigma$ versus electronic temperature $T$ for various standard deviations $\Delta \mu$ of the chemical potential according to Eq. (3). (b) Effect of convoluting the Fermi function $f_{\mu T}$ with a Gaussian distribution $p_{\mu}$ of the chemical potential. The resulting $\left\langle f_{\mu T}\right\rangle$ is much less temperature dependent. (c, d) Heat-induced change in conductivity and dielectric function as calculated from Eq. (3) and $\operatorname{Re} \sigma_{\infty}$ for several electronic temperatures $T$. Note the good qualitative agreement with the experimental data in Figs. 2(d) and 2(e).

2(e) for various pump-probe delays $\tau$. Transient transmission measurements with various pump-photon energies indicated that roughly half of the absorbed $1.6 \mathrm{eV}$ photons excite the small-gap and metallic NTs [17]. Within 0.2 ps, the excited electrons relax to a Fermi-Dirac distribution $f_{\mu T}$ with electronic temperature $T$ [32]. In the schematic of Fig. 1(a), this leads to an increased number of electrons and holes around the Fermi level. They partially block interband transitions and so reduce the FIR absorption, in agreement with the observed negative $\operatorname{Re} \Delta \sigma_{\tau}$ in Fig. 2(d). In other words, the signal is induced by the change in electron temperature $T$ via the change $F_{\mu T G}-F_{\mu T_{\text {amb }} G}$ in the occupation numbers. Equation (3) and the measured equilibrium conductivity can now be used to model the pumpinduced conductivity change as $\operatorname{Re} \Delta \sigma(\omega) / \operatorname{Re} \sigma_{\infty}(\omega)=$ $\left\langle F_{\mu T G}\right\rangle /\left\langle F_{\mu T_{\text {amb }} G}\right\rangle-1$ where $G=\hbar \omega$. Comparing the modeled results of Figs. 3(c) and 3(d) to the experimental data of Figs. 2(d) and 2(e) shows that our simple model reproduces the experimental results well for a suitable choice of the instantaneous electronic temperature $T(\tau)$. The decay of $T$ with the pump-probe delay $\tau$ reflects the cooling of the electrons on a picosecond time scale [33].

Increasing the pump fluence leads to higher electronic temperatures following sample excitation. Accordingly, the sample absorption should decrease further as is indeed verified by our data in Fig. 2(f). However, this trend levels off at fluences above $\approx 200 \mu \mathrm{J} \mathrm{cm}^{-2}$, which is easily understood by Eq. (2): The occupation-number difference $F_{\mu=0, T, G}$ becomes insensitive to temperature $T$ when $k_{\mathrm{B}} T$ is much larger than the transition energy $G$.

In summary, we have presented a simple model based on an ensemble of two-level systems which explains all ob- served qualitative features of the equilibrium and nonequilibrium FIR conductivity of small-gap NT ensembles. In particular, the controversially discussed weak temperature dependence of the FIR conductivity is consistent with the tube-to-tube variation of the chemical potential, which is $\approx 0.1 \mathrm{eV}$ in our sample. Our results suggest to use the FIR conductivity as a sensitive and contact-free probe of the doping of NT ensembles as well as an ultrafast "thermometer" of the NT electrons [33].

L. P. acknowledges support from a Marie Curie program and C. M. A. from FQRNT (Québec). Funding was provided by the DFG through SFB 450, the RQMP (Québec), and the CRC Program.

[1] M.S. Dresselhaus et al., Carbon Nanotubes: Synthesis, Structure, and Applications (Springer, New York, 2001).

[2] M. Ouyang et al., Science 292, 702 (2001).

[3] P. Delaney et al., Nature (London) 391, 466 (1998).

[4] E. S. Snow et al., Science 307, 1942 (2005).

[5] O. J. Korovyanko et al., Phys. Rev. Lett. 92, 017403 (2004).

[6] F. Wang et al., Science 308, 838 (2005).

[7] L. Perfetti et al., Phys. Rev. Lett. 96, 027401 (2006).

[8] A. Ugawa, A. G. Rinzler, and D. B. Tanner, Phys. Rev. B 60, R11305 (1999).

[9] T.-I. Jeon et al., J. Appl. Phys. 95, 5736 (2004).

[10] N. Akima et al., Adv. Mater. 18, 1166 (2006).

[11] K. Kamarás et al., Science 301, 1501 (2003).

[12] B. Ruzicka et al., Phys. Rev. B 61, R2468 (2000).

[13] F. Borondics et al., Phys. Rev. B 74, 045431 (2006).

[14] M. E. Itkis et al., Nano Lett. 2, 155 (2002).

[15] T. Kampfrath et al., Phys. Status Solidi B 244, 3950 (2007).

[16] A. Hagen and T. Hertel, Nano Lett. 3, 383 (2003).

[17] R. J. Ellingson et al., Phys. Rev. B 71, 115444 (2005).

[18] R. Huber et al., Appl. Phys. Lett. 76, 3191 (2000).

[19] Q. Wu et al., Appl. Phys. Lett. 70, 1784 (1997).

[20] T. Kampfrath et al., Appl. Phys. Lett. 90, 231113 (2007).

[21] U. J. Kim et al., Phys. Rev. Lett. 95, 157402 (2005).

[22] T. W. Noh, P. H. Song, and A. J. Sievers, Phys. Rev. B 44, 5459 (1991).

[23] P. Parkinson et al., Nano Lett. 7, 2162 (2007).

[24] H. Ehrenreich et al., Phys. Rev. 115, 786 (1959).

[25] R. von Baltz et al., Phys. Status Solidi B 51, 499 (1972).

[26] F. L. Shyu et al., J. Phys. Soc. Jpn. 71, 1820 (2002).

[27] We neglect homogeneous broadening due to optical dephasing; it can be included by a convolution with an appropriate line shape function. See, for example, R.W. Boyd, Nonlinear Optics (Academic Press, San Diego, 1992).

[28] For $G \lesssim k_{\mathrm{B}} T$, the effect of $\mu$ and $\Delta \mu$ on $\operatorname{Re} \sigma$ is seen by linearizing $F_{\mu T G}$ around $\overline{G=0} 0$ in Eq. (3) which yields $\operatorname{Re} \sigma \propto \exp \left(-2 \mu^{2} / A^{2}\right) / A$ with $A=\sqrt{2\left(k_{\mathrm{B}} T\right)^{2}+\Delta \mu^{2}}$.

[29] C. Zhou, J. Kong, and H. Dai, Phys. Rev. Lett. 84, 5604 (2000).

[30] A. Das et al., Phys. Rev. Lett. 99, 136803 (2007).

[31] S. Suzuki et al., Appl. Phys. Lett. 85, 127 (2004).

[32] A. Hagen et al., Appl. Phys. A 78, 1137 (2004).

[33] T. Kampfrath et al., Phys. Rev. Lett. 95, 187403 (2005). 